\section{Sintomáticos respiratórios nas unidades de atenção primária no Município de Vitória, Espírito Santo, Brasil}

\author{
Respiratory symptomatics among patients at \\ primary health clinics in Vitória, Espírito Santo \\ State, Brazil
}

\author{
1 Programa de Pós-graduação \\ em Saúde Coletiva, \\ Universidade Federal do \\ Espírito Santo, Vitória, Brasil. \\ 2 Secretaria Municipal de \\ Saúde de Vitória, Vitória, \\ Brasil. \\ 3 Núcleo de Doenças \\ Infecciosas, Universidade \\ Federal do Espírito Santo, \\ Vitória, Brasil. \\ Correspondência \\ E. L. N. Maciel \\ Programa de Pós-graduação \\ em Saúde Coletiva \\ Universidade Federal do \\ Espírito Santo. \\ Av. Marechal Campos 1468 \\ Vitoria, ES \\ 29040-091, Brasil. \\ emaciel@ndi.ufes.br
}

\section{Abstract}

The aim of this study was to estimate the rate of positive respiratory symptomatics among patients seeking treatment at primary health clinics in Vitória, Espirito Santo State, Brazil. This cross-sectional study included 603 individuals that answered a questionnaire with socio-demographic data and a question on cough in the previous three weeks. The prevalence ratio was calculated with 5\% significance. The proportion of patients with positive respiratory symptoms in the municipality was 4\%, varying from 1.6 to $11.7 \%$ between regions. The majority (71\%) did not report cough as the reason for coming to the health clinic. Variables significantly associated with respiratory symptoms were: shortness of breath (PR $=6.29 ; 95 \% C I: 2.22-21.81)$ and lack of appetite ( $P R=2.75$; 95\%CI: 1.08-6.82). Among patients with respiratory symptoms, cough was the principal reason for seeking treatment. This condition was only associated with two variables, thus demonstrating the need to adopt different strategies to identify these individuals among the daily patient flow in primary health clinics.

Tuberculosis; Respiratory Signs and Symptoms; Primary Health Care
Claudia Maria Marques Moreira 1,2

Eliana Zandonade 1

Thamy Lacerda 1

Ethel Leonor Noia Maciel 1,3
A tuberculose (TB) foi responsável pela maioria das mortes no fim do século XIX e início do século XX, e ainda permanece como a principal causa de óbito entre as doenças infecciosas entre adultos no mundo inteiro ${ }^{1}$. A Organização Mundial da Saúde (OMS) estima que aproximadamente um terço da população mundial se encontra infectada pelo Mycobacterium tuberculosis, bacilo causador da doença, causando um total de 8,8 milhões de casos novos e a ocorrência de 1,6 milhão de óbitos anualmente 2 .

O Município de Vitória (Espírito Santo) é um dos 315 municípios brasileiros considerados prioritários no controle da $\mathrm{TB}$, em razão de critérios adotados pelo Programa Nacional de Controle da Tuberculose (PNCT) ${ }^{3}$, sendo responsável por aproximadamente $30 \%$ das notificações do estado. No ano de 2003, foram notificados 158 casos de TB de todas as formas e 85 casos da forma pulmonar bacilífera, correspondendo à taxa de incidência de 50,8 e 27,7/100 mil habitantes, respectivamente, valores acima da média nacional e estadual no mesmo ano 4; e estudo da distribuição espacial de casos novos de TB no município mostrou distribuição desigual das taxas de incidência entre as Regiões de Saúde 5 .

A tosse é um sintoma relacionado a várias enfermidades, descrita como uma das causas mais freqüentes de busca por atendimento médico. Associada à expectoração, pode ser o primeiro 
sinal de tuberculose pulmonar, que é a principal forma clínica responsável pela transmissão da doença 6 . Para que se diagnostiquem precocemente os casos de TB existentes, é necessária a busca de casos por meio da identificação do sintomático respiratório definido como: indivíduo maior de 15 anos com tosse produtiva há mais de três semanas 7. Todas as pessoas com essa sintomatologia devem ser identificadas e submetidas ao exame de baciloscopia de escarro o mais precocemente possível 7,8. A busca de casos suspeitos, o atendimento ao paciente desde o diagnóstico ao tratamento, não significa somente uma ação individual e sim uma ação de saúde coletiva 9 , pois elimina as fontes de contágio na população.

O controle da TB é definido como prioridade entre as políticas de saúde no Brasil com ações a serem realizadas em todos os níveis de complexidade do SUS. Entre as ações da Atenção Primária, estão a busca ativa de casos, diagnóstico, acompanhamento e tratamento dos pacientes sob supervisão quando indicado 7 .

Em municípios prioritários no Estado de São Paulo, onde se observou tendência à descentralização das ações do Programa de Controle da Tuberculose (PCT) para as unidades básicas de saúde (UBS), a descoberta dos sintomáticos respiratórios ainda é realizada por meio de campanhas e na demanda espontânea das unidades 10 . Em muitas situações, a busca ativa dos sintomáticos respiratórios ocorre na investigação dos contatos de algum caso novo detectado, sendo a tosse um sintoma não merecedor de ação imediata, não recebendo atenção das equipes de saúde num primeiro contato 11 . Estudo realizado em Vitória mostrou que os agentes comunitários de saúde (ACS) descreveram como sintomas da TB a febre, seguida por emagrecimento, e a tosse prolongada foi o sintoma de menor relevância para eles na identificação de suspeitos de TB 12 .

O Ministério da Saúde estima um percentual de $5 \%$ de sintomáticos respiratórios entre os consultantes de primeira vez, maiores de 15 anos nas UBS 7. Estudos realizados para conhecer a proporção de sintomáticos respiratórios e as diferenças existentes nos níveis locais da assistência à saúde da população são importantes, visto que servem de parâmetros para o planejamento das ações operacionais do PCT no município.

O presente estudo tem como objetivo estimar a proporção de sintomáticos respiratórios na população maior de 15 anos, que busca atendimento nas Unidades de Atenção Primária do Município de Vitória.

\section{Métodos}

A cidade de Vitória, capital do Estado do Espírito Santo, apresenta uma população de 314.042 habitantes 13 , integrando-se a uma área geográfica de grande nível de urbanização composta pela Região Metropolitana de Vitória com outros cinco municípios no seu entorno. A cidade contemplou diferentes formas de ocupação ao longo dos últimos 50 anos. Desde uma área com edificações verticais, com população referindo mais de 15 anos de estudos, a uma área de periferia, onde predomina a construção de casas de alvenaria improvisadas e de madeira, abrigando população sócio-economicamente desfavorecida, de baixa renda e com $11,3 \%$ de analfabetismo 13

O Município de Vitória habilitou-se na gestão plena da Atenção Básica em 28 de janeiro de 1998, agregando também serviços da rede complementar. Seu modelo de atenção foi baseado na idéia de Sistemas Locais de Saúde, iniciando na época, a implantação do Programa Saúde da Família (PSF) e Programa de Agentes Comunitários de Saúde (PACS) como estratégia de reorganização da Atenção Básica 14. Atualmente, o município apresenta cerca de $70 \%$ da população cobertos pelo PSF/PACS. O município é dividido em seis Regiões de Saúde com a rede ambulatorial distribuída em 28 unidades de saúde, com as seguintes características: 20 unidades de saúde da família (USF), 4 unidades com PACS e 4 unidades de saúde tradicionais (UBS). Além da rede básica, existem 2 unidades de pronto atendimento e 6 centros de referência.

\section{Delineamento do estudo e tamanho da amostra}

Estudo seccional, descritivo, cuja população de interesse foi definida como indivíduos maiores de 15 anos de idade, de ambos os sexos, que se dirigiram as UBS para atendimento médico individual. Seu tamanho foi determinado tomandose como base o número de consultas de primeira vez realizadas nas unidades de saúde do município, que foram 600 mil. Durante quatro meses (período estimado da realização da coleta de dados), esperar-se-ia uma população de 200 mil.

Para se calcular o tamanho amostral $n$ capaz de fornecer uma estimativa com precisão $d$ da proporção $p$ de indivíduos sintomáticos respiratórios, utilizou-se a seguinte fórmula:

$$
n=\frac{N^{*} z^{2} * p^{*}(1-p)}{d^{2} *(N-1)+z^{2} *(1-p)}
$$

onde,

$N$ : população total;

$z$ : valor $z$ (corresponde ao nível de significância); 
$d$ : precisão em valor absoluto;

$p$ : prevalência esperada.

O tamanho final da amostra foi dado por $n_{\text {final }}=n^{*}$ (efeito do desenho). $\mathrm{O}$ efeito do desenho é uma maneira de aumentar o tamanho amostral, para que a amostra seja capaz de captar a correlação/dependência que existe entre os indivíduos, devido ao fato de eles serem selecionados em uma mesma unidade de saúde. Apesar de propor uma amostragem aleatória estratificada (Regiões de Saúde) por cotas e utilizar a fórmula de amostra aleatória simples para o cálculo, o efeito do desenho também serve para corrigir uma diferença no tamanho amostral (correlações introduzidas pelos conglomerados, que são as unidades de saúde dentro de cada região). No presente estudo, consideramos o efeito do desenho igual a 2 .

Valendo-se dessa população de interesse e utilizando o programa Epi Info 6.04 (Centers for Disease Control and Prevention, Atlanta, Estados Unidos), o tamanho amostral foi calculado até o nível de significância de $5 \%$, com prevalência esperada de $5 \%$ e precisão desejada de $2,5 \%$. O tamanho de amostra mínimo foi de 292 indivíduos. Com o efeito do desenho, igual a 2, o tamanho amostral encontrado foi de 584 indivíduos.

Como são 28 UBS e elas estão divididas em seis regiões, foi realizada uma amostragem aleatória estratificada por cotas. Cada Região de
Saúde foi considerada como um estrato, sendo sorteadas três unidades, dividindo-se proporcionalmente o número de entrevistados para cada uma (Tabela 1). As entrevistas foram feitas pela manhã em uma sala reservada. Os pacientes foram selecionados aleatoriamente da lista dos atendimentos agendados para o dia.

\section{Definições de termos}

Neste estudo, foi definido como sintomático respiratório o indivíduo maior de 15 anos com tosse produtiva há três semanas ou mais.

\section{Coleta de dados}

A coleta dos dados foi realizada no período de 11 de fevereiro a 9 de maio de 2008. O instrumento utilizado foi um questionário de perguntas fechadas sobre dados sócio-demográficos, motivo da consulta e averiguação da possibilidade de o individuo ser sintomático respiratório por meio de pergunta direta sobre a presença de tosse há mais de três semanas. As entrevistas foram realizadas pela pesquisadora e por duas enfermeiras com domínio teórico da doença, no horário habitual de atendimento das unidades de saúde, durante o período necessário para se alcançar o número de entrevistados definido na amostra.

Tabela 1

Distribuição do total amostral, por unidade de saúde e por Região de Saúde. Município de Vitória, Espírito Santo, Brasil, 2008.

\begin{tabular}{|c|c|c|}
\hline Região & Unidades sorteadas & Questionários por unidade \\
\hline \multirow[t]{3}{*}{ Maruípe } & Bairro da Penha & 42 \\
\hline & Consolação & 42 \\
\hline & Santa Martha & 42 \\
\hline \multirow[t]{3}{*}{ Centro } & Fonte Grande & 35 \\
\hline & Avelina & 35 \\
\hline & Santa Teresa & 35 \\
\hline \multirow[t]{3}{*}{ Santo Antônio } & Santo Antonio & 20 \\
\hline & Alagoano & 20 \\
\hline & Grande Vitória & 20 \\
\hline \multirow[t]{3}{*}{ São Pedro } & São Pedro V & 27 \\
\hline & Santo André & 27 \\
\hline & Ilha Caieiras & 27 \\
\hline \multirow[t]{3}{*}{ Forte São João } & Jesus Nazaré & 35 \\
\hline & Forte São João & 35 \\
\hline & Ilha Santa Maria & 35 \\
\hline \multirow[t]{3}{*}{ Continental } & Jardim da Penha & 42 \\
\hline & Jabour & 42 \\
\hline & Bairro República & 42 \\
\hline Total & 18 & 603 \\
\hline
\end{tabular}




\section{Análise dos dados}

Os dados foram apresentados por Região de Saúde, sob forma de freqüências absolutas e relativas de cada variável categórica. Para as variáveis quantitativas, utilizou-se a média e o desvio padrão. Para o cálculo da proporção de sintomáticos respiratórios na amostra, foi calculada a prevalência entre os indivíduos que apresentaram tosse há três semanas ou mais sobre o total da amostra. Para avaliar diferenças entre a condição de sintomático respiratório e não sintomático respiratório, em relação às variáveis "sexo, hábitos (tabagismo e uso de álcool), e sintomas (emagrecimento, febre, falta de ar, falta de apetite)", foi calculada a razão de prevalência com respectivo intervalo de 95\% de confiança (IC95\%). O teste t para amostras independentes foi usado para a variável média de idade. Para a variável "anos de estudo”, foi realizado teste não paramétrico para tendência linear. Os resultados foram analisados usando o pacote estatístico SPSS, versão 13.0 (SPSS Inc., Chicago, Estados Unidos).

Apesar de não ser objetivo do trabalho a estimação da incidência da positividade para a doença, foi solicitada a baciloscopia de escarro do individuo que se enquadrou na condição de sintomático respiratório. A pesquisa foi realizada de acordo com as recomendações da Resolução $n^{o}$. 196, de 10 de outubro de 1996, do Conselho Nacional de Saúde para pesquisa científica em seres humanos, aprovada pelo Comitê de Ética da Universidade Federal do Espírito Santo sob o no. 044/07 e pela Secretaria de Saúde (SEMUS) do Município de Vitória.

\section{Resultados}

Independentemente do perfil demográfico de cada Região de Saúde, que não foi alvo deste estudo, observou-se que as características dos usuários entrevistados foram semelhantes. A maioria dos indivíduos foi do sexo feminino, com média de idade de 41,5 anos. Em relação ao número de anos estudados, a maioria referiu de 5 a 8 anos. A maioria das pessoas entrevistadas residia no território de abrangência da unidade e não era profissional da área da saúde. A referência ao uso de cigarros e de álcool esteve presente em percentual semelhante (Tabela 2 ).

As queixas ligadas ao aparelho respiratório corresponderam a $11,3 \%$ do total dos atendimentos. Cento e sessenta e quatro entrevistados $(27,2 \%)$ referiram tosse. Dentre eles, 80 indivíduos $(13,3 \%)$ referiram tosse há menos de uma semana, 52 pessoas $(8,6 \%)$ relataram tosse há uma semana, 8 pessoas $(1,3 \%)$ referiram tosse há duas semanas. Foram encontrados 24 indivíduos correspondendo à proporção de 4\% (IC95\%: 2,56$5,86)$, que referiram tosse há três semanas ou mais, considerados sintomáticos respiratórios distribuídos de forma desigual entre as Regiões de Saúde (Tabela 3). Dos sintomáticos respiratórios encontrados, nove colheram pelo menos uma amostra de escarro, todas negativas.

Dos 164 entrevistados que relataram tosse, 35 (21,3\%) o fizeram espontaneamente, como motivo que os levou a procurar a unidade de saúde, e $129(78,7 \%)$ só relataram a tosse quando perguntados diretamente. Tal situação foi semelhante em todas as regiões do município (Tabela 4).

No cálculo da razão de prevalência, foi encontrada diferença significativa entre a condição de sintomático respiratório e não sintomático respiratório em relação ao relato de falta de ar (RP: 6,29; IC95\%: 2,22-21,81) e relato de falta de apetite (RP: 2,75; IC95\%: 1,08-6,82). Não foram verificadas diferenças em relação ao sexo, referência atual a tabagismo e uso de álcool, relato de emagrecimento e febre (Tabela 5). O teste t para amostras independentes para a média da idade obteve valor de $\mathrm{p}=0,84$, não significativo. Para a variável anos de estudo, o teste não paramétrico para tendência linear com valor de $\mathrm{p}=0,73$, também não mostrou diferença significativa (dados não mostrados).

\section{Discussão}

Em estudo realizado no México, onde foram encontrados 3,6\% de sintomáticos respiratórios entre os consultantes, observou-se a necessidade de atenção para a busca do sintoma tosse entre os usuários que buscassem o serviço de saúde por qualquer queixa e a redução do tempo de tosse para menos de uma semana para diagnóstico de casos de TB ${ }^{15}$. Na Venezuela, foram encontrados 4,46\% de sintomáticos respiratórios, percentual próximo ao nosso estudo, entre os consultantes por queixas respiratórias. Mais de $40 \%$ dos casos de TB pulmonar encontrados referiram tosse com menos de 15 dias de duração, mostrando a dificuldade de se fixar o tempo de evolução da tosse para se definir o termo sintomático respiratório. Atribuiu-se este fato à possível dificuldade dos indivíduos em se recordarem precisamente da data do início do sintoma 16. Em serviços de Atenção Primária na Índia, foram encontrados $2,5 \%$ de sintomáticos respiratórios (tosse há três semanas ou mais), com incremento para $4,2 \%$ quando se considerou o tempo de tosse a partir de duas semanas 17 .

No Município de Fortaleza (Ceará) 18, observou-se que o percentual de sintomáticos respi- 
Distribuição das características da população da amostra, por Região de Saúde. Município de Vitória, Espírito Santo, Brasil, 2008.

\begin{tabular}{|c|c|c|c|c|c|c|c|}
\hline & $\begin{array}{c}\text { Município } \\
\text { de Vitória } \\
\text { n (\%) }\end{array}$ & $\begin{array}{c}\text { São Pedro } \\
\text { n (\%) }\end{array}$ & Santo Antonio & $\begin{array}{c}\text { Forte } \\
\text { São João } \\
\text { n (\%) }\end{array}$ & $\begin{array}{l}\text { Centro } \\
\mathrm{n}(\%)\end{array}$ & $\begin{array}{l}\text { Maruípe } \\
\text { n (\%) }\end{array}$ & $\begin{array}{l}\text { Continental } \\
\text { n (\%) }\end{array}$ \\
\hline \multicolumn{8}{|l|}{ Sexo } \\
\hline Masculino & $175(29,0)$ & $22(27,2)$ & $15(25,0)$ & $33(31,4)$ & $36(34,3)$ & $42(33,3)$ & $27(21,4)$ \\
\hline Feminino & $428(71,0)$ & $59(72,8)$ & $45(75,0)$ & $72(68,6)$ & $69(65,7)$ & $84(66,7)$ & $99(78,6)$ \\
\hline \multicolumn{8}{|c|}{ Número de anos estudados } \\
\hline Nenhum & $62(10,3)$ & $11(13,6)$ & $5(8,3)$ & $14(13,3)$ & $14(13,3)$ & $11(8,7)$ & $7(5,6)$ \\
\hline $1-4$ & $122(20,2)$ & $16(19,8)$ & $14(23,3)$ & $19(18,1)$ & $22(21,0)$ & $28(22,2)$ & $23(18,3)$ \\
\hline $5-8$ & $204(33,8)$ & $28(34,6)$ & $22(36,7)$ & $34(32,4)$ & $32(30,5)$ & $42(33,3)$ & $46(36,5)$ \\
\hline $9-11$ & $191(31,7)$ & $25(30,9)$ & $17(28,3)$ & $35(33,3)$ & $33(31,4)$ & $40(31,7)$ & $41(32,5)$ \\
\hline $11 \mathrm{e}+$ & $24(4,0)$ & $1(1,2)$ & $2(3,3)$ & $3(2,9)$ & $4(3,8)$ & $5(4,0)$ & $9(7,1)$ \\
\hline \multicolumn{8}{|c|}{ Ocupação na área da saúde } \\
\hline Não & $583(96,7)$ & $81(100,0)$ & $60(100,0)$ & $103(98,1)$ & $99(94,3)$ & $122(96,8)$ & $118(93,7)$ \\
\hline \multicolumn{8}{|l|}{ Reside no território } \\
\hline Sim & $567(94,0)$ & $80(98,8)$ & $59(98,3)$ & $100(95,2)$ & $97(92,4)$ & $121(96,0)$ & $110(87,3)$ \\
\hline \multicolumn{8}{|l|}{ Uso de cigarros } \\
\hline Sim & $97(16,1)$ & $12(14,8)$ & $5(8,3)$ & $19(18,1)$ & $19(18,1)$ & $25(19,8)$ & $17(13,5)$ \\
\hline \multicolumn{8}{|l|}{ Uso de álcool } \\
\hline $\operatorname{Sim}$ & $98(16,3)$ & $10(12,3)$ & $11(18,3)$ & $17(16,2)$ & $24(22,9)$ & $23(18,3)$ & $13(10,3)$ \\
\hline Total (N) & 603 & 81 & 60 & 105 & 105 & 126 & 126 \\
\hline $\begin{array}{l}\text { Média de idade } \pm \\
\text { desvio padrão }\end{array}$ & $41,5 \pm 17,5$ & $40,2 \pm 17,8$ & $42,5 \pm 17,4$ & $42,5 \pm 17,1$ & $44,9 \pm 17,2$ & $36,3 \pm 14,7$ & $44,9 \pm 17,7$ \\
\hline
\end{tabular}

Tabela 3

Distribuição da amostra, segundo tempo de tosse referido, por Região de Saúde. Município de Vitória, Espírito Santo, Brasil, 2008.

\begin{tabular}{|c|c|c|c|c|c|c|c|}
\hline & $\begin{array}{c}\text { Município } \\
\text { de Vitória } \\
\text { n (\%) }\end{array}$ & São Pedro & Santo Antonio & $\begin{array}{c}\text { Forte } \\
\text { São João } \\
\text { n (\%) }\end{array}$ & $\begin{array}{l}\text { Centro } \\
\text { n (\%) }\end{array}$ & $\begin{array}{l}\text { Maruípe } \\
\text { n (\%) }\end{array}$ & $\begin{array}{c}\text { Continental } \\
\text { n (\%) }\end{array}$ \\
\hline Não referiu tosse & $439(72,8)$ & $52(64,3)$ & $41(68,3)$ & $59(56,2)$ & $85(81,0)$ & $87(69,0)$ & $115(91,2)$ \\
\hline Tosse há < 1 semana & $80(13,3)$ & $12(14,8)$ & $7(11,7)$ & $26(24,7)$ & $13(12,3)$ & $16(12,7)$ & $6(4,8)$ \\
\hline Tosse há 1 semana & $52(8,6)$ & $13(16,0)$ & $5(8,3)$ & $13(12,4)$ & $3(2,9)$ & $16(12,7)$ & $2(1,6)$ \\
\hline Tosse há 2 semanas & $8(1,3)$ & $1(1,2)$ & $0(0,0)$ & $3(2,9)$ & $1(0,9)$ & $2(1,6)$ & $1(0,8)$ \\
\hline Tosse há $\geq 3$ semanas & $24(4,0)$ & $3(3,7)$ & $7(11,7)$ & $4(3,8)$ & $3(2,9)$ & $5(4,0)$ & $2(1,6)$ \\
\hline Total & $603(100,0)$ & $81(100,0)$ & $60(100,0)$ & $105(100,0)$ & $105(100,0)$ & $126(100,0)$ & $126(100,0)$ \\
\hline
\end{tabular}

ratórios foi variável entre os serviços de saúde, mantendo-se em média, em torno de $6,5 \%$ no município. Dos pacientes que referiram tosse há três semanas ou mais na entrevista, cerca de $30 \%$ não haviam procurado o serviço de saúde pelos sintomas respiratórios, semelhante ao Município de Vitória.
No Rio de Janeiro, estudando-se a prevalência de casos de TB entre sintomáticos respiratórios que buscaram atendimento em unidade de saúde, foram encontrados 10,7\% de usuários com tosse há mais de uma semana, e entre esses, uma prevalência de TB pulmonar de $2,7 \%$. Não houve associação com o tempo referido de tosse, 
Distribuição da amostra, segundo referência ao sintoma tosse de qualquer duração, por Região de Saúde. Município de Vitória, Espírito Santo, Brasil, 2008.

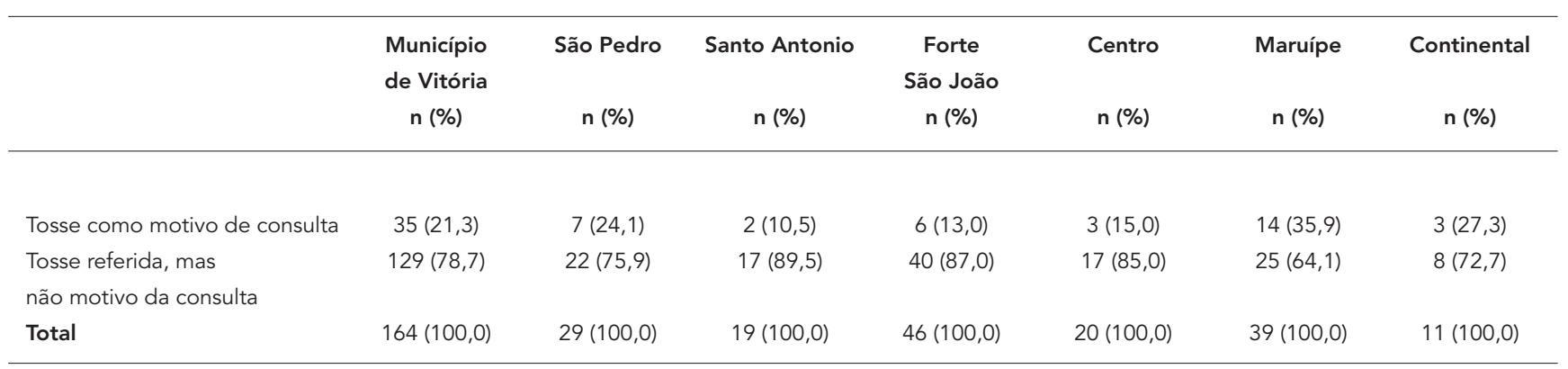

Tabela 5

Distribuição de características da amostra, segundo condição de sintomático respiratório e não sintomático respiratório. Município de Vitória, Espírito Santo, Brasil, 2008.

\begin{tabular}{|c|c|c|c|}
\hline Variáveis & $\begin{array}{c}\text { Sintomático } \\
\text { respiratório } \\
\mathrm{n}(\%)\end{array}$ & $\begin{array}{c}\text { Não sintomático } \\
\text { respiratório } \\
n(\%)\end{array}$ & RP (IC95\%) \\
\hline Sexo & & & $1,24(0,4-3,1)$ \\
\hline Masculino & $8(33,3)$ & $166(28,7)$ & \\
\hline Feminino & $16(66,7)$ & $413(71,3)$ & \\
\hline Presença de tabagismo & $6(25,0)$ & $91(15,7)$ & $1,78(0,56-4,86)$ \\
\hline Presença de uso de álcool & $4(16,7)$ & $94(16,2)$ & $1,03(0,25-3,18)$ \\
\hline Emagrecimento & $8(33,3)$ & $116(20,0)$ & $1,99(0,72-5,08)$ \\
\hline Febre & $1(29,2)$ & $94(16,2)$ & $0,22(0,005-1,41)$ \\
\hline Falta de ar & $19(79,2)$ & $218(37,7)$ & $6,29(2,22-21,81)$ * \\
\hline Falta de apetite & $11(45,8)$ & $136(23,5)$ & $2,75(1,08-6,82)$ ** \\
\hline
\end{tabular}

IC95\%: intervalo de 95\% de confiança; RP: razão de prevalência.

* $\mathrm{p}=0,0001$

** $p=0,025$.

sugerindo que em locais com alta prevalência da doença, o diagnóstico de casos de TB seria incrementado se o tempo de três semanas fosse reduzido 19 .

Em Curitiba (Paraná), estudo realizado com uma amostra semelhante ao nosso estudo, encontrou-se uma prevalência de 2,9\% de sintomáticos respiratórios 20 . No Município de Belém (Pará), foi encontrado um percentual de 10,3\% de sintomáticos respiratórios entre os consultantes de UBS 21 e $66 \%$ dos sintomáticos respiratórios encontrados pelos pesquisadores não haviam sido identificados pelos profissionais de saúde.

Em nosso estudo, a maioria dos sintomáticos respiratórios $(71 \%)$ não havia procurado a unidade de saúde por causa da tosse. Além disso, não se encontraram diferenças estatísticas nas maiorias das variáveis, de modo que o sintomático respiratório pode ser qualquer usuário que busque o sistema de saúde. Tal dado demonstra a necessidade de capacitação dos profissionais na identificação desses indivíduos presentes na rotina diária das unidades de saúde.

Uma das limitações deste estudo foi o fato de que, nos indivíduos que realizaram as baciloscopias, os resultados foram negativos, indicando que a estratégia de buscar os sintomáticos respiratórios é pouco específica, não sendo possível com base nestes achados mostrar a sensibilidade da estratégia.

No entanto, a detecção passiva, ou seja, identificação do sintomático respiratório somente quando este chega à unidade de saúde para pro- 
cura espontânea do diagnóstico, pode não levar à descoberta de todos os casos de TB existentes na população, pois a tosse, quando prolongada, pode não ser percebida como sintoma que leve o indivíduo a buscar o serviço de Atenção Primária. É necessário, portanto, incluir a investigação de sintomáticos respiratórios nas avaliações de rotina de pacientes que procuram os serviços de saúde, sobretudo em áreas com alta prevalência de TB.

Outro importante ponto que merece ser destacado é a observação da distribuição desigual dos sintomáticos respiratórios entre as Regiões de Saúde, semelhante ao estudo da distribuição espacial dos casos novos de TB no município 5. $\mathrm{O}$ fato pode estar relacionado às diferenças na transmissão da doença dentro do município, onde taxas de incidência superiores foram identificadas nos locais em que também se encontrou maior incidência de sintomáticos respiratórios. Esses resultados sugerem que a matriz programática do Ministério da Saúde 7 deveria ser reorientada de acordo com a prevalência da doença nos bairros e não no município como um todo, uma vez que diferenças intramunicipais devem ser levadas em consideração. Torna-se necessária a adoção de diferentes estratégias com atenção ao sintoma tosse entre os indivíduos, sob forma de detecção passiva e busca ativa, contribuindo dessa forma para o diagnóstico precoce e o controle da doença no nível dos bairros adstritos pelas unidades de saúde.

\section{Resumo}

O objetivo do estudo foi estimar a proporção de sintomáticos respiratórios na população que busca atendimento nas unidades de atenção primária do Município de Vitória, Espírito Santo. Foi realizado um estudo transversal do qual participaram 603 indivíduos que responderam a um questionário referente a dados sócio-demográficos e questão sobre tosse há mais de três semanas. Foi calculada a razão de prevalência com nível de significância de 5\%. A proporção de sintomáticos respiratórios encontrada no município foi de $4 \%$, variando de 1,6 a 11,7\% entre as regiões. A maioria (71\%) não referiu a tosse como motivo de procura do serviço. As variáveis significativamente associadas à condição de sintomático respiratório foram: falta de ar $(R P=$ 6,29; IC95\%: 2,22-21,81) e falta de apetite $(R P=2,75$; IC95\%: 1,08-6,82). Entre os sintomáticos respiratórios, a tosse foi o principal motivo de consulta. Observou-se associação dessa condição com somente duas variáveis, demonstrando a necessidade de adoção de diferentes estratégias para busca e identificação desses indivíduos na demanda diária das unidades de atenção primária.

Tuberculose; Sinais e Sintomas Respiratórios; Atenção Primária à Saúde

\section{Colaboradores}

C. M. M. Moreira participou da revisão de literatura, elaboração da metodologia, coleta, análise e interpretação dos resultados e aprovação da versão final. E. Zandonade participou de todas as etapas desta pesquisa, elaboração da metodologia, análise e revisão do texto final. T. Lacerda colaborou na coleta, análise dos dados, revisão bibliográfica e redação final. E. L. N. Maciel participou de todas as etapas da pesquisa e da revisão crítica do texto final.

\section{Agradecimentos}

Este estudo recebeu apoio financeiro através do Edital MCT/CNPq/MS-SCTIE-DECIT 25/2006 - Estudo de Doenças Negligenciadas e do International Clinical, Operational and Health Services Research and Trainning Award, Edital ICOHRTA 5U2R TW006883-02. 


\section{Referências}

1. Ducati RG, Ruffino-Netto A, Bassp LA, Santos DS. The resumption of consumption - a review on tuberculosis. Mem Inst Oswaldo Cruz 2006; 11:697714.

2. World Health Organization. Global tuberculosis control: surveillance, planning, financing. http://www.who.int/tb/publications/glob al_report/2007/pdf/bra.pdf (acessado em 15/ Mai/2007).

3. Secretaria de Vigilância em Saúde, Ministério da Saúde. Programa Nacional de Controle da Tuberculose. Brasília: Ministério da Saúde; 2004.

4. Ministério da Saúde. Saúde Brasil 2005: uma análise da situação de saúde. Brasília: Ministério da Saúde; 2005.

5. Vieira RCA, Prado TN, Siqueira MG, Dietze R, Maciel ELN. Distribuição espacial dos casos novos de tuberculose em Vitória, Estado do Espírito Santo, no período entre 2000 e 2005. Rev Soc Bras Med Trop 2008; 41:82-6.

6. Sociedade Brasileira de Pneumologia e Tisiologia. II diretrizes brasileiras no manejo da tosse crônica. J Bras Pneumol 2006; 32 Suppl 6:403-46.

7. Ministério da Saúde. Tuberculose: guia de vigilância epidemiológica. Brasília: Ministério da Saúde; 2002.

8. World Health Organization. Training modules: managing TB at the raion level. Identification of tuberculosis suspects. http://whqlibdoc.who.int/ hq/2003/WHO_CDS_TB_2002.310_mod2_eng.pdf (acessado em 15/Mai/2007).

9. Nagpaul DR. La búsqueda de casos de tuberculosis: una perspectiva. Rev Argent Tuberc Enfermedades Pulm Salud Pública 1985; 46:5-11.

10. Oliveira MF. A busca de sintomáticos respiratórios nos municípios prioritários para o controle da tuberculose do Estado de São Paulo (2005) [Dissertação de Mestrado]. Ribeirão Preto: Escola de Enfermagem de Ribeirão Preto, Universidade de São Paulo; 2006.

11. Muniz JN, Palha PF, Monroe AA, Gonzales RC, Ruffino-Netto A, Villa TCS. A incorporação da busca ativa de sintomáticos respiratórios para o controle da tuberculose na prática do agente comunitário de saúde. Ciênc Saúde Coletiva 2005; 10:315-21.
12. Maciel ELN, Vieira RCA, Milani EC, Brasil M, Fregona G, Dietze R. O agente comunitário de saúde no controle da tuberculose: conhecimento e percepções. Cad Saúde Pública 2008; 24:1377-86.

13. Secretaria de Desenvolvimento da Cidade, Prefeitura de Vitória. Vitória on line. Histórico dos bairros. http://www.vitoria.es.gov.br (acessado em 01/ Set/2007)

14. Secretaria Municipal de Saúde. Manual para a Atenção Básica do Município de Vitória: primeira versão. Vitória: Secretaria Municipal de Saúde; 2000.

15. Marín MAV, Cholula CT, Castillo RO. Tuberculosis pulmonar entre sintomáticos respiratorios detectados en las unidades de salud de la SSA, en el estado de Tlaxcala, México. Rev Inst Nac Enfermedades Respir 1999; 12:29-34.

16. Armengol R, Machado CB, Quiñones LR. Encuesta de sintomáticos respiratorios en establecimientos de salud de la zona metropolitana de Caracas. Gac Méd Caracas 1992; 100:121-7.

17. Santha T, Garg R, Subramani R, Chandrasekaran V, Selvakumar N, Sisodia RS, et al. Comparison of cough of 2 and 3 weeks to improve detection of smear-positive tuberculosis cases among out-patients in India. Int J Tuberc Lung Dis 2005; 9:61-8.

18. Mota MRT. Prevalência de sintomáticos respiratórios nos consultantes de primeira vez no Município de Fortaleza. Bol Pneumol Sanit 2004; 12:85-90

19. Bastos LGV, Fonseca LS, Mello FCQ, Ruffino-Netto A, Golub JL, Conde MB. Prevalence of pulmonary tuberculosis among respiratory symptomatic subjects in an out-patient primary health unit. Int J Tuberc Lung Dis 2007; 11:156-60.

20. Gabardo BMA, Rossoni AMO, Costa HMLM, Schichta BS, Schlichting GCB, Gomes DC, et al. Prevalência de sintomáticos respiratórios em Unidades de Saúde. In: Anais do III Encontro Nacional de Tuberculose. Brasília: Sociedade Brasileira de Pneumologia e Tisiologia; 2008. p. R21.

21. Rodrigues ILA, Cardoso NC. Avaliação da detecção de sintomáticos respiratórios em serviços de saúde da rede pública do Município de Belém - Pará. In: Anais do III Encontro Nacional de Tuberculose. Brasília: Sociedade Brasileira de Pneumologia e Tisiologia; 2008. p. R33.

Recebido em 25/Abr/2009

Aprovado em 29/Jun/2010 